\title{
Transoral Laser Surgery for Supraglottic Carcinoma
}

Jong Chul Hong ${ }^{1}$

Kang Dae Lee ${ }^{2}$

\footnotetext{
${ }^{1}$ Department of Otolaryngology-Head and Neck Surgery, Dong-A University College of Medicine, Busan, Korea

${ }^{2}$ Department of Otolaryngology-Head and Neck Surgery, Kosin University College of Medicine, Busan, Korea
}

\begin{abstract}
Primary treatment modalities of supraglottic cancer usually consist of single or combined methods. Treatment protocols include single transoral surgery, external transcervical approach, and radiation therapy or a combination of these methods. The objective of surgery for supraglottic cancer is cure of the tumor, with preservation of voice, deglutition, and an intact airway. Transoral laser surgery is found to achieve comparable oncologic results with open surgery. Moreover, the complication rate was lower in laser surgery compared to the conventional open approaches and there were no major complications. Transoral laser supraglottic laryngectomy seems to be a feasible and oncologically safe procedure in the treatment of fresh or recurrent supraglottic cancer ranging from $\mathrm{T} 1$ to selected $\mathrm{T} 3$ with minimal preepiglottic space involvement.
\end{abstract}

\section{Key words}

Laser; Supraglottis; Carcinoma

\section{Correspondence}

Kang Dae Lee

Department of Otolaryngology-Head and Neck Surgery, Kosin University College of Medicine, 34, Amnam-dong, Seo-gu, Busan 602-715, Korea Tel: +82-51-990-6248

Fax: +82-51-245-8539

E-mail: kosinentdyahoo.co.kr

(C) Korean Society for Laser Medicine and Surgery

(c) This is an open access article distributed under the terms of the Creative Commons Attribution NonCommercial License (http://creativecommons.org/ licenses/by-nc/3.0) which permits unrestricted noncommercial use, distribution, and reproduction in any medium, provided the original work is properly cited. 


\section{INTRODUCTION}

Primary treatment modalities of supraglottic cancer are defined as single or combined methods. Treatment protocols include single transoral surgery, external transcervical approach and radiation therapy or the combination of these. The aims of surgery for supraglottic cancer are cure of the tumor with preservation of voice, deglutition, and an intact airway. Organ conservation surgery for supraglottic cancer includes open partial laryngectomy and transoral laser surgery. In the last three decades, the use of transoral laser suregry has been increasingly popularized as a feasible approach to Tis, T1, T2, and selected T3 supraglottic cancer (preepiglottic space invasion). ${ }^{1}$ The oncologic results of transoral laser surgery seem to be comparable to those of classic supraglottic laryngectomy if clean surgical margins can be reached. ${ }^{2}$

Vaughan from Boston was the first surgeon describing a supraglottic partial laryngectomy carried out transorally with a carbon dioxide laser in 1978. ${ }^{3}$ Since 1979 Steiner used the carbon dioxide laser for transoral endoscopic
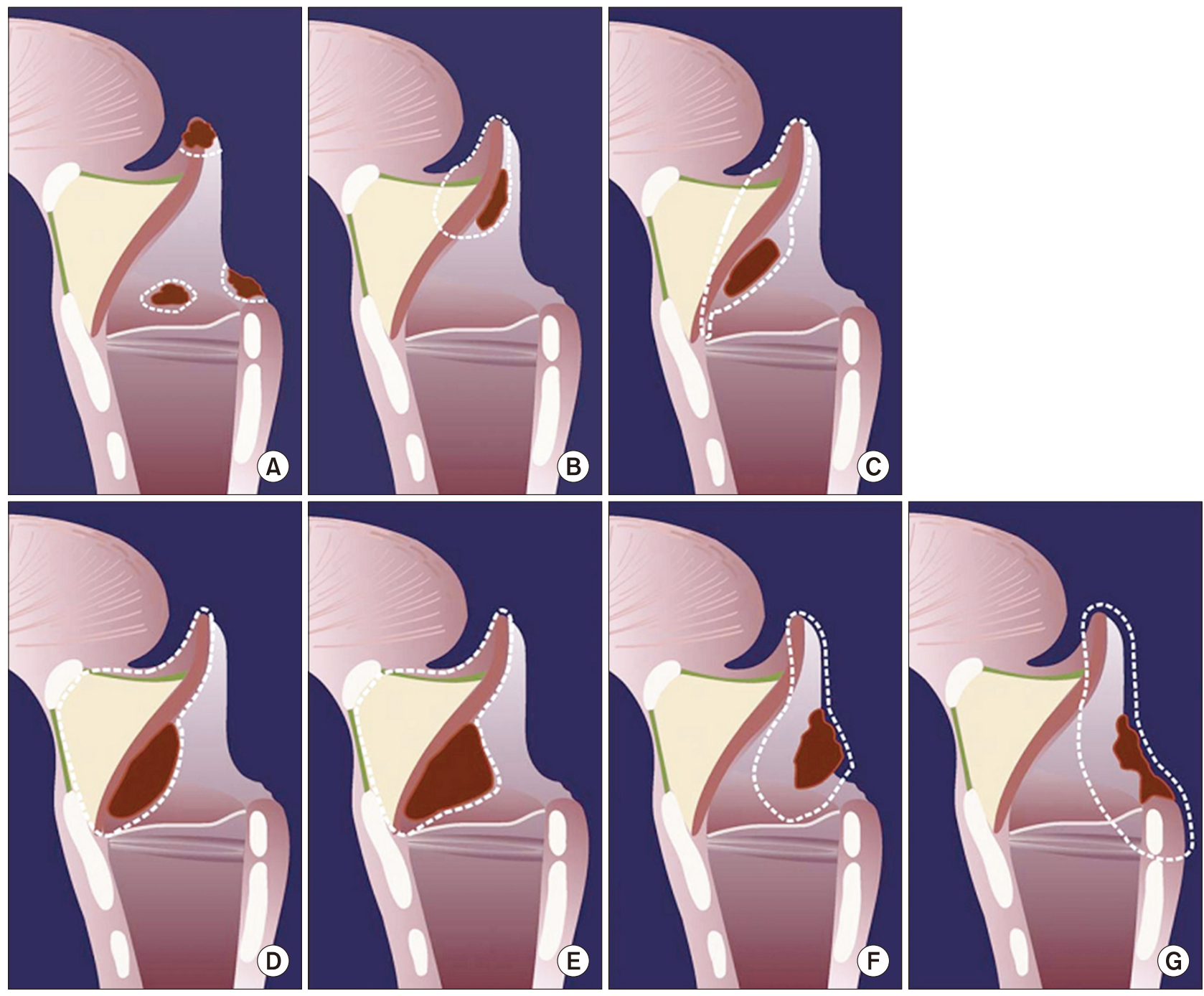

Fig. 1. The classification of the endoscopic supraglottic partial laryngectomy.10 (A) Type I - Limited excision. (B) Type IIa - Resection includes half of the suprahyoid epiglottis. (C) Type IIb - Total epiglottectomy, The pharyngo-epiglottic, ary-epiglottic and ventricular folds are preserved. (D) Type IIIa - Resection of T1-T2 tumors extending to the petiole of the epiglottis must include the pre-epiglottic space. (E) Type IIIb - Ventricular folds can be completely dissected from the thyroid cartilage along the inner surface towards the ventricle of Morgagni. (F) Type IVa - Resection includes the free edge of the epiglottis, the threefolds' region (the lateral free edge of the epiglottis, the aryepiglottic fold and the pharyngoepiglottic fold with possible extension to the ventricular folds). (G) Type IVb - Resection includes the arytenoid. The resection may include the inner or medial and anterior part of the pyriform sinus. 
surgery of selected supraglottic carcinomas. ${ }^{4,5}$ In 1983 , Davis et al. reported on a first series of 20 patients with benign bulky lesions obstructing the airway or small suprahyoid carcinomas of the epiglottis that were treated with laser epiglottectomy. ${ }^{6}$ Later Davis et al. and Zeitels et al. published the results of laser microsurgery in selected patients with supraglottic carcinomas. T1 and T2 as well as selected $\mathrm{T} 3$ and $\mathrm{T} 4$ carcinomas were resected with this method. ${ }^{7.8}$ Recently, Hong and Lee et al. reported good oncologic and functional outcomes of salvage transoral laser supraglottic laryngectomy after radiation failure.

In 2009, the European Laryngological Society is proposing a classification of the different laryngeal endoscopic supraglottic partial laryngectomies. This classification comprises four types of supraglottic laryngectomies (Fig. 1): ${ }^{10}$

Type I: Limited excision

Excision of small superficial tumors on the free border of the epiglottis, the aryepiglottic fold, the arytenoids or the ventricular fold or any other part of the supraglottis

Type II: Medial supraglottic laryngectomy with partial resection of the pre-epiglottic space

Type Ila: Resection includes half of the suprahyoid epiglottis

Type IIb: Total epiglottectomy, The pharyngo-epiglottic, ary-epiglottic and ventricular folds are preserved

Type III: Medial supraglottic laryngectomy with resection of the pre-epiglottic space

Type IIla: Resection of T1-T2 tumors extending to the petiole of the epiglottis must include the pre-epiglottic space
Type IIlb: Ventricular folds can be completely dissected from the thyroid cartilage along the inner surface towards the ventricle of Morgagni

Type IV: Lateral supraglottic laryngectomy

Type IVa: Resection includes the free edge of the epiglottis, the threefolds' region(the lateral free edge of the epiglottis, the ary-epiglottic fold and the pharyngoepiglottic fold with possible extension to the ventricular folds

Type IVb: Resection includes the arytenoid. The resection may include the inner or medial and anterior part of the pyriform sinus

\section{OPERATIVE TECHNIQUE IN KOSIN GOSPEL HOSPITAL}

Preoperative staging of the tumor is carried out by suspension laryngoscopy and computed tomography (CT) of the neck to evaluate preepiglottic space, paraglottic space and lymph node involvement. The patients underwent general anesthesia. We used tubed (closed) laryngoscope and distensible laryngoscope to provide a sufficient exposure of the larynx (Fig. 2). The surgeries were performed using a Sharplan $\mathrm{CO}_{2}$ laser (Sharplan Model 30C/40C, Lumenis, Tel Aviv, Israell coupled with a micromanipulator (AcuSpot 715), set to an output power of 2-4 W with continuous mode. The operator made a clinical assessment of the margins of resection with the operating microscope lOPMI VARIO/S88, Carl Zeiss, German), rigid $30^{\circ}$ and $70^{\circ}$ endoscopes. Small tumors were excised in en-bloc. Large tumors were
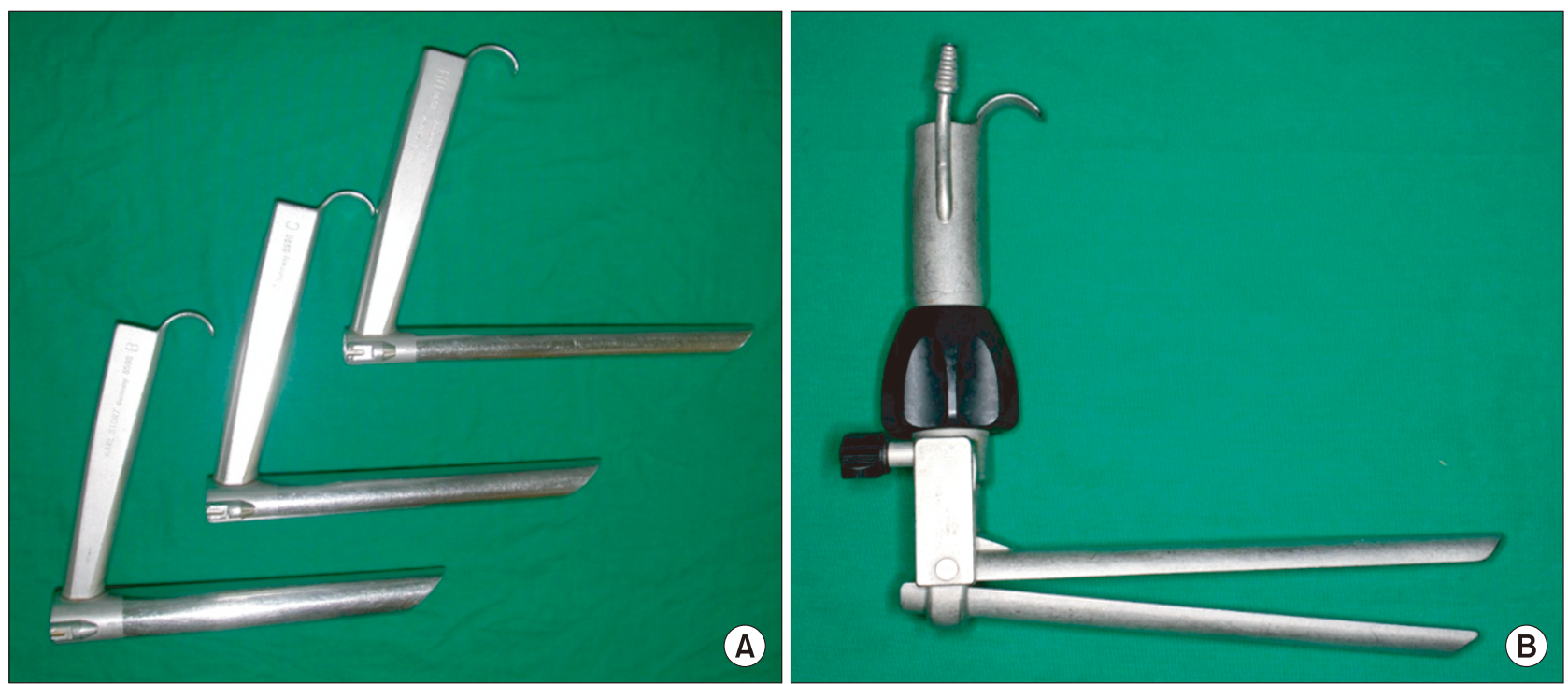

Fig. 2. Laryngoscope. (A) Tubed (closed) laryngoscope, (B) Distensible laryngoscope. 


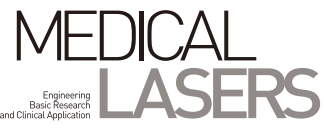

excised with piecemeal resection. Minor bleeding was controlled by coagulation forceps and suction bovie. To reduce postoperative bleeding, the branches of superior laryngeal artery were clipped with hemoclips prior to cutting (Fig. 3). All specimens were sent for frozen biopsy to get free margins. If positive or suspicious margin was reported, further resection was conducted. After complete excision of the tumor, the wounds were always left open to secondary healing.

\section{Neck dissection}

In supraglottic cancer, nodal spread is very frequent and plays a valuable role in prognosis; so, treatment of the neck must be performed by surgery, radiotherapy or both. In open procedures, neck dissection is performed in the same surgical act, but there is yet controversy for transoral laser surgery. Classically, authors prefer to defer neck treatment and execute it as a secondstage procedure to minimize postoperative edema and the need for a tracheotomy, ${ }^{11,12}$ but others have previously demonstrated that transoral laser surgery and simultaneous neck dissection are safe as a single-stage procedure, making recovery time shorter and assisting in early start of other therapies when needed. ${ }^{9,13,14}$

\section{Tracheotomy}

One of the advantages of transoral laser surgery is lower tracheotomy rate than open surgery. In Hong et al report, they conducted tracheotomy in $86 \%$ patients and decannulation was possible in all patients without any other complications. ${ }^{9}$ The reasons why they did tracheotomy: (1) adequate exposure of surgical field especially in radiation-induced mucosal edema, (2) management of postoperative mucosal swelling because of extensive laser resection, (3) management of postoperative upper airway swelling after simultaneous neck dissection, (4) management of postoperative aspiration or bleeding. Tracheotomy is familiar to the otolaryngologist, it is simple procedure with minimal complications. The incidence of postoperative hemorrhage is similar in both endoscopic and open approaches and ranges from $3 \%$ to $10 \%$. 15,16 However, endolaryngeal bleeding in endoscopically treated, non tracheotomized patients is a serious and potentially lethal complication, not only because of the blood loss but also because of possible aspiration of blood, and hypoxia. ${ }^{17}$
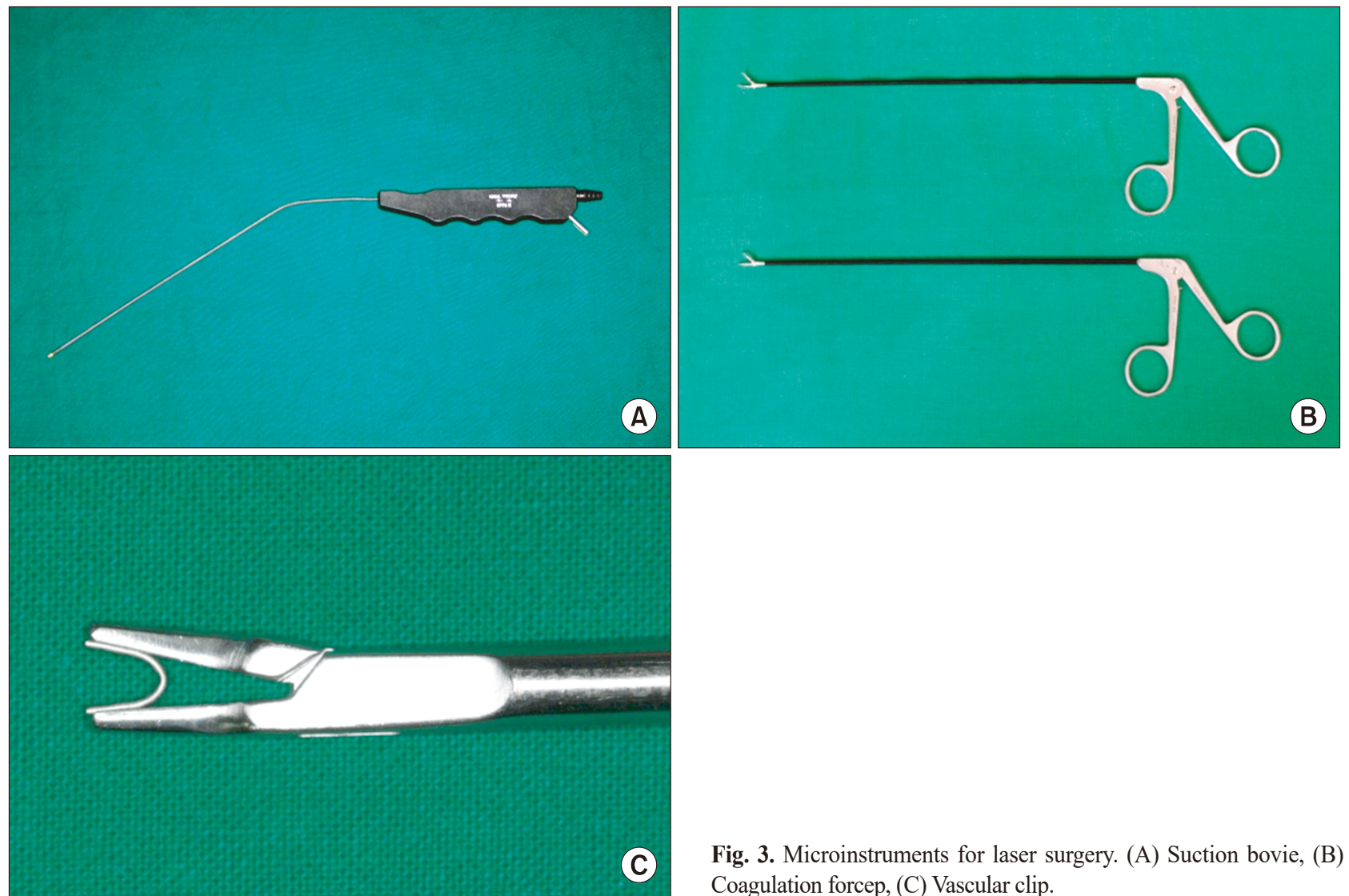

Fig. 3. Microinstruments for laser surgery. (A) Suction bovie, (B) Coagulation forcep, (C) Vascular clip. 
The author recommend tracheotomy in case of extensive laser resection or simultaneous neck dissection.

\section{Complications}

The most frequent is aspiration, which was reported by Kollisch et al. with an incidence of $11.5 \%{ }^{18}$ But it is known that incidence of aspiration pneumonia is variable and also depends on the patient's condition, including age and motivation. ${ }^{15,16,19}$ The incidence of hemorrhage oscillated between 3 and $14 \%$. ${ }^{12,15,16,20}$

The incidence of postoperative hemorrhage is about equal for open supraglottic laryngectomy, SCPL-CHP, and laser microsurgery with an average between 3-14\% in different studies. ${ }^{12,15,20,21}$ However, every postoperative bleeding after endoscopic surgery in a non-tracheotomized patient is a serious and potentially life-threatening complication due to the risk of aspiration of blood. ${ }^{22}$

\section{Oncologic result}

Zeitels et al. analyzed the results of laser microsurgery in 42 patients with supraglottic carcinomas in a multiinstitutional study. ${ }^{23}$ Nineteen patients with T1 or T2 tumors treated with laser microsurgery alone were cured. Of 23 patients, mostly with T2 tumors, who received both surgery and postoperative irradiation, 4 developed local recurrences and were salvaged by total laryngectomy. In 1997, Eckel reported the results of supraglottic laser resection in 46 patients with T1 and T2 tumors. Four (8.7\%) patients developed local or locoregional recurrences, 5 (10.9\%) required secondary laryngectomy due to functional reasons. ${ }^{24}$ Iro et al. reported in 1998 on transoral supraglottic laser resections in 141 patients with the following UICC stage distribution: stage I, 23.4\%; stage II, 25.5\%; stage III, 16.3\%; and stage IV, 34.8\%. ${ }^{25}$

Ambrosch et al. treated 50 patients with pT3 supraglottic laryngeal carcinomas (40 stage III, 10 stage IV) with transoral laser microsurgery. ${ }^{26}$ In 41 (82\%) patients, the tumor was classified pT3 due to invasion of the preepiglottic fat; in $9(18 \%)$ patients vocal cord fixation was present preoperatively. In 13 (26\%) patients invasion of the paraglottic space and in $9(18 \%)$ cases superficial tumor spread to one or both vocal cords was diagnosed. These patients were treated with laser microsurgery as an alternative to extended supraglottic laryngectomy, SCPL-CHP, or total laryngectomy. The 5 -year local control rate was $86 \%$. Four per cent of the patients underwent total laryngectomy due to local tumor recurrence. The 5-year recurrence-free survival rate was $71 \%$. Peretti et al. ${ }^{27}$ reported on a series of 80 patients treated with laser microsurgery in which 20 patients with pT3 lesions showing "limited infiltration" of the preepiglottic fat. In $88.2 \%$ of patients larynx preservation was achieved, the 5 -year disease-free survival was $59.6 \%$. Hong et al. reported salvage transoral laser surgery after radiation failure cases. They showed 2 and 5 -year overall survival rates were $85.7 \%$ and $68.6 \%$, respectively by using Kaplan-Meier method. The ultimate local control rate is $100 \%$ and laryngeal preservation rate is $85.7 \%$.

\section{Functional result}

All authors who have reported on the transoral laser resection of supraglottic carcinomas agree that swallowing rehabilitation proceeds more quickly and has better outcomes than open supraglottic laryngectomy. The rate of secondary laryngectomy for persistent aspiration after open supraglottic laryngectomy is in the range of $3.5-12.5 \%{ }^{28-31}$ The incidence of postoperative aspiration and the time needed for swallowing rehabilitation varies with age, general health condition, and with the extent of resection of the base of tongue and arytenoid cartilage. ${ }^{21,32,33}$ Due to considerable morbidity and postoperative functional impairment, open supraglottic laryngectomy often does not qualify as a treatment option, particularly in elderly patients with pre-existing pulmonary disease. On the whole, the functional results of laser microsurgery for supraglottic T1-T3 carcinomas are very favorable. Patients need nasogastric tube feeding normally for a few postoperative days. Aspiration is minimal and occurs in the early postoperative period only and there is a reduced need for secondary tracheostomy and laryngectomy due to aspiration problems. ${ }^{15,16,20,25,26,34}$ Mechanisms contributing significantly to the recovery of swallowing function after supraglottic, either open or endoscopic laryngectomy are: the oropharyngeal transit time of the bolus, closure of the airway at the laryngeal entrance, the position of the laryngeal remnant in relation to the base of the tongue, and the movement of the base of the tongue toward the posterior pharyngeal wall. ${ }^{33,35,36}$ Patients who regain these functions postoperatively meet the prerequisites for a normal swallowing function.

We attribute the early and consistently successful swallowing rehabilitation in the patients treated by us to a variety of factors. The avoidance of tracheotomy, the integrity of the base of the tongue and pharyngeal muscles and the preservation of the hyoid bone with the supra and infrahyoid muscles, enable the larynx to move normally during deglutition. At least one mobile arytenoids cartilage was preserved in all operations, to enable functional closure of the larynx. We also believe 
that preserving the extralaryngeal portions of the superior laryngeal nerves is an important factor in sensory reinnervation.

Nasogastric tube or percutaneous endoscopic gastrostomy was not necessary in any patients. All started soft diet within 1-3 days after surgery. One patient had a postoperative aspiration pneumonia and successfully treated by adequate antibiotic therapy. Unilateral vocal cord fixation occured in 2 patients. In these cases, laser resection was extended to ipsilateral cricoarytenoid joint or thyroarytenoid muscle. But no additional treatment such as a laryngoplasty was necessary. We did not observe any of the following complications such as postoperative bleeding, delayed upper airway stenosis, granuloma, and pharyngocutaneous fistula did not occur.

\section{CONCLUSIONS}

Transoral laser surgery seems to achieve comparable oncologic results with open surgery. Moreover, laser surgery showed less complication rate than conventional open approaches and no major complications. Transoral laser supraglottic laryngectomy seems a feasible and oncologically safe procedure in fresh or recurrent supraglottic cancer of ranging from T1 to selected T3 for minimal preepiglottic space involvement.

\section{REFERENCES}

1. Laccourreye 0 , Weinstein G, Naudo P, Cauchois R, Laccourreye H, Brasnu D. Supracricoid partial laryngectomy after failed laryngeal radiation therapy. Laryngoscope 1996;106:495-8.

2. Spriano G, Pellini R, Romano G, Muscatello L, Roselli R. Supracricoid partial laryngectomy as salvage surgery after radiation failure. Head Neck 2002;24:759-65.

3. Vaughan CW. Transoral laryngeal surgery using the CO2 laser: laboratory experiments and clinical experience. Laryngoscope 1978;88:1399-420.

4. Steiner W, Jaumann MP, Pesch HJ. Endoskopische Therapie von Krebsfrühstadien im Larynx - vorläufige Ergebnisse. Arch Otorhinolaryngol 1981;231:637-60.

5. Steiner W. Experience in endoscopic laser surgery of malignant tumours of the upper aero-digestive tract. Adv Otorhinolaryngol 1988;39:135-44.

6. Davis RK, Shapshay SM, Strong MS, Hyams VJ. Transoral partial supraglottic resection using the CO2 laser. Laryngoscope 1983;93:429-32.

7. Davis RK, Kelly SM, Hayes J. Endoscopic CO2 laser excisional biopsy of early supraglottic cancer. Laryngoscope 1991;101:6803.
8. Zeitels SM, Vaughan CW, Domanowski GF. Endoscopic management of early supraglottic cancer. Ann Otol Rhinol Laryngol 1990;99:951-6.

9. Hong JC, Kim SW, Lee HS, Han YJ, Park HS, Lee KD. Salvage transoral laser supraglottic laryngectomy after radiation failure: a report of seven cases. Ann Otol Rhinol Laryngol 2013; 122:85-90.

10. Remacle M, Hantzakos A, Eckel H, Evrard AS, Bradley PJ, Chevalier D, et al. Endoscopic supraglottic laryngectomy: a proposal for a classification by the working committee on nomenclature, European Laryngological Society. Eur Arch Otorhinolaryngol 2009;266:993-8.

11. Steiner W, Ambrosch P. Endoscopic Laser Surgery of the Upper Aerodigestive Tract: With Special Emphasis on Cancer Surgery. Stuttgart: Thieme; 2000.

12. Rudert HH, Werner JA, Höft S. Transoral carbon dioxide laser resection of supraglottic carcinoma. Ann Otol Rhinol Laryngol 1999;108:819-27.

13. Rodrigo JP, Charlone R, Cabanillas R, Llorente JL, Suárez C. Is it necessary to perform neck dissections as a staged procedure in laser supraglottic laryngectomy? Acta Otorrinolaringol Esp 2008;59:345-8.

14. Rigby MH, Taylor SM. Review of transoral laser microsurgery for cancer of the upper aerodigestive tract. J Otolaryngol Head Neck Surg 2011;40:113-21.

15. Cabanillas R, Rodrigo JP, Llorente JL, Suárez V, Ortega P, Suárez C. Functional outcomes of transoral laser surgery of supraglottic carcinoma compared with a transcervical approach. Head Neck 2004;26:653-9.

16. Peretti G, Piazza C, Cattaneo A, De Benedetto L, Martin E, Nicolai $P$. Comparison of functional outcomes after endoscopic versus open-neck supraglottic laryngectomies. Ann Otol Rhinol Laryngol 2006;115:827-32.

17. Rodrigo JP, Suárez C, Silver CE, Rinaldo A, Ambrosch P, Fagan JJ, et al. Transoral laser surgery for supraglottic cancer. Head Neck 2008;30:658-66.

18. Köllisch M, Werner JA, Lippert BM, Rudert H. Functional results following partial supraglottic resection. Comparison of conventional surgery vs. transoral laser microsurgery. Adv Otorhinolaryngol 1995;49:237-40.

19. Csanády M, Czigner J, Vass G, Jóri J. Transoral CO2 laser management for selected supraglottic tumors and neck dissection. Eur Arch Otorhinolaryngol 2011;268:1181-6.

20. Ambrosch P, Kron M, Steiner W. Carbon dioxide laser microsurgery for early supraglottic carcinoma. Ann Otol Rhinol Laryngol 1998; 107:680-8.

21. Hirano M, Kurita S, Tateishi M, Matsuoka H. Deglutition following supraglottic horizontal laryngectomy. Ann Otol Rhinol Laryngol 1987;96:7-11.

22. Kremer B, Schlöndorff $G$. Late lethal secondary hemorrhage 
after laser supraglottic laryngectomy. Arch Otolaryngol Head Neck Surg 2001;127:203-5.

23. Zeitels SM, Koufman JA, Davis RK, Vaughan CW. Endoscopic treatment of supraglottic and hypopharynx cancer. Laryngoscope 1994;104:71-8

24. Eckel HE. Endoscopic laser resection of supraglottic carcinoma. Otolaryngol Head Neck Surg 1997;117:681-7.

25. Iro H, Waldfahrer F, Altendorf-Hofmann A, Weidenbecher M, Sauer R, Steiner W. Transoral laser surgery of supraglottic cancer: follow-up of 141 patients. Arch Otolaryngol Head Neck Surg 1998;124:1245-50.

26. Ambrosch P, Rödel R, Kron M, Steiner W. Die transorale Lasermikrochirurgie des Larynxkarzinoms. Eine retrospective Analyse von 657 Patientenverläufen. Onkologe 2001;7:505-12.

27. Peretti G, Piazza C, Ansarin M, De Benedetto L, Cocco D, Cattaneo A, et al. Transoral CO2 laser microsurgery for Tis-T3 supraglottic squamous cell carcinomas. Eur Arch Otorhinolaryngol 2010;267:1735-42.

28. Prades JM, Simon PG, Timoshenko AP, Dumollard JM, Schmitt T, Martin C. Extended and standard supraglottic laryngectomies: a review of 110 patients. Eur Arch Otorhinolaryngol 2005;262: 947-52.

29. Suarez C, Rodrigo JP, Herranz J, Diaz C, Fernandez JA. Complications of supraglottic laryngectomy for carcinomas of the supraglottis and the base of the tongue. Clin Otolaryngol Allied Sci 1996;21:87-90.

30. Bocca E, Pignataro O, Oldini C. Supraglottic laryngectomy: 30 years of experience. Ann Otol Rhinol Laryngol 1983;92:14-8.

31. Sevilla MA, Rodrigo JP, Llorente JL, Cabanillas R, López F, Suárez C. Supraglottic laryngectomy: analysis of 267 cases. Eur Arch Otorhinolaryngol 2008;265:11-6.

32. Beckhardt RN, Murray JG, Ford CN, Grossman JE, Brandenburg $\mathrm{JH}$. Factors influencing functional outcome in supraglottic laryngectomy. Head Neck 1994;16:232-9.

33. Logemann JA, Gibbons P, Rademaker AW, Pauloski BR, Kahrilas PJ, Bacon M, et al. Mechanisms of recovery of swallow after supraglottic laryngectomy. J Speech Hear Res 1994;37:96574.

34. Roh JL, Kim DH, Park Cl. Voice, swallowing and quality of life in patients after transoral laser surgery for supraglottic carcinoma. J Surg Oncol 2008;98:184-9.

35. Rademaker AW, Logemann JA, Pauloski BR, Bowman JB, Lazarus CL, Sisson GA, et al. Recovery of postoperative swallowing in patients undergoing partial laryngectomy. Head Neck 1993;15:325-34.

36. Schweinfurth JM, Silver SM. Patterns of swallowing after supraglottic laryngectomy. Laryngoscope 2000;110:1266-70. 